\title{
Lamotrigine overdose cause skin rash and angioedema*
}

\author{
Mohammad Alkayem $^{1 \#}$, Hussein Assallum ${ }^{2}$ \\ Department of Internal Medicine, Lincoln Medical and Mental Health Center, New York, USA \\ Email: "mo54434@hotmail.com
}

Received 9 April 2013; revised 9 May 2013; accepted 16 May 2013

Copyright (c) 2013 Mohammad Alkayem, Hussein Assallum. This is an open access article distributed under the Creative Commons Attribution License, which permits unrestricted use, distribution, and reproduction in any medium, provided the original work is properly cited.

\begin{abstract}
A 23 years old female presented to Lincoln Medical and Mental Health center with skin rash and angioedema after she received 20 pills of lamotrigine $25 \mathrm{mg}$, and the patient used to take this medication before two tables a day for many months, after she received the appropriate management she improved. After reviewing MEDLINE we found a few cases reported life threatening complications related to lamotrigine intoxication and sudden increase the dose, so we should be aware about these complications before prescribing this medication.
\end{abstract}

Keywords: Lamotrigine; Over Dose; Angioedema

\section{INTRODUCTION}

To describe the physical manifestations of lamotrigine toxicity presenting as skin rash and angioedema.

\section{CASE REPORT}

A 23 years old Hispanic female presented to Lincoln Medical and Mental Health Center with itchy rash affecting her face, body, and swelling of her eyelids and lower lip, after taking approximately 20 pills of lamotrigine in an apparent suicide attempt. She denies SOB, cough, fever, palpitation, headache or blurry vision. She has history of bipolar disorder and used to be on lamotrigine $25 \mathrm{mg}$ bid for many months. No other significant medical history. No history of allergies. Patient uses Alcohol daily and Marijuana sometimes. Physical exam show mild edema in eyes lids, few erythematous

\footnotetext{
"Guarantor of Submission: The corresponding author is the guarantor of submission.

Author's Contributions: Mohammad Alkayem, MD: first author, wrote whole the case, took the consent and the Images, prepared the article for publication and sent it to OJIM. Hussein Assallum, MD: second author, reviewed and revised the article.

Conflict of Interest: Null.

${ }^{\#}$ Corresponding author.
}

edematous papules on cheeks, bilateral upper extremities (Figures 1 and 2), lower extremities, chest, abdomen and back. The rest of physical exam was normal. Blood work normal, urine toxicology was positive for Marijuana. EKG showed prolonged QT interval (Figure 3). Steroids and anti-histamine started which improved her symptoms.

Dermatology assessment: Urticarial with angioedema, may be related to lamotrigine overdose.

\section{DISCUSSION}

In our case, the patient developed mild angioedema and urticaria, 12 - 24 hours, after she took approximately 500 $\mathrm{mg}$ of lamotrigine. Lamotrigine is a new antiepileptic medication approved by the US Food and Drug Administration as adjunctive treatment of partial seizures in adults and children, as well as for adjunctive therapy for primary generalized tonic-clonic seizures and Lennox-Gastaut syndrome [1,2]. It is also used as first line medication for bipolar disorder maintenance treatment [3]. Common side effects of lamotrigine at regular dose include nausea, vomiting, chest pain, peripheral edema, insomnia, somnolence, impaired coordination, dizziness, ataxia, irritability, rash, dermatitis, dysmenorrhea, increased libido, dyspepsia, nystagmus, abnormal vision, and in rare cases have been reported to cause multiorgan hypersensitivity reactions, life-threatening skin rashes, CNS depression, aseptic meningitis [4-7]. Overdose of lamotrigine usually causes drowsiness, lethargy, nausea, vomiting, ataxia, dizziness, tachycardia, coma, respiratory depression. There is report of a case in which lamotrigine caused anticonvulsant hypersensitivity syndrome [8,9]. The incidence of urticaria, periorbital and lip edema, immediately after Lamotrigine overdose in our patient, made us suspect overdose as the cause of the symptoms.

\section{CONCLUSION}

Lamotrigine overdose may manifest as angioedema, and 


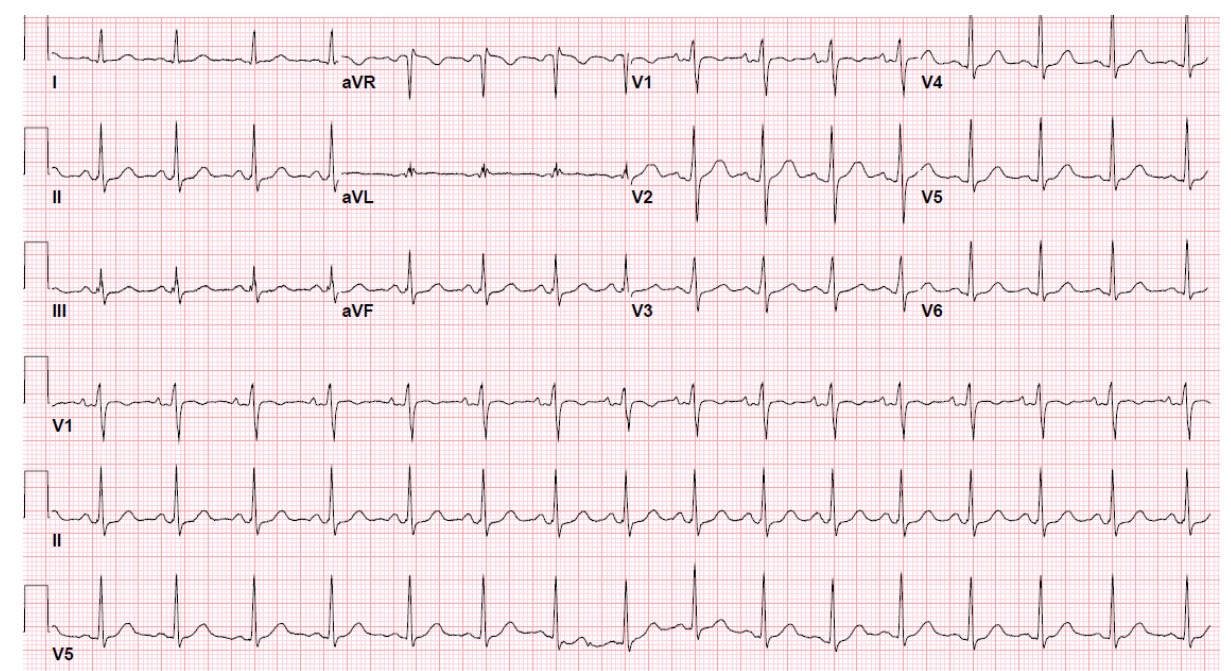

Figure 1. EKG show sinus tachycardia with prolong QT intervals.

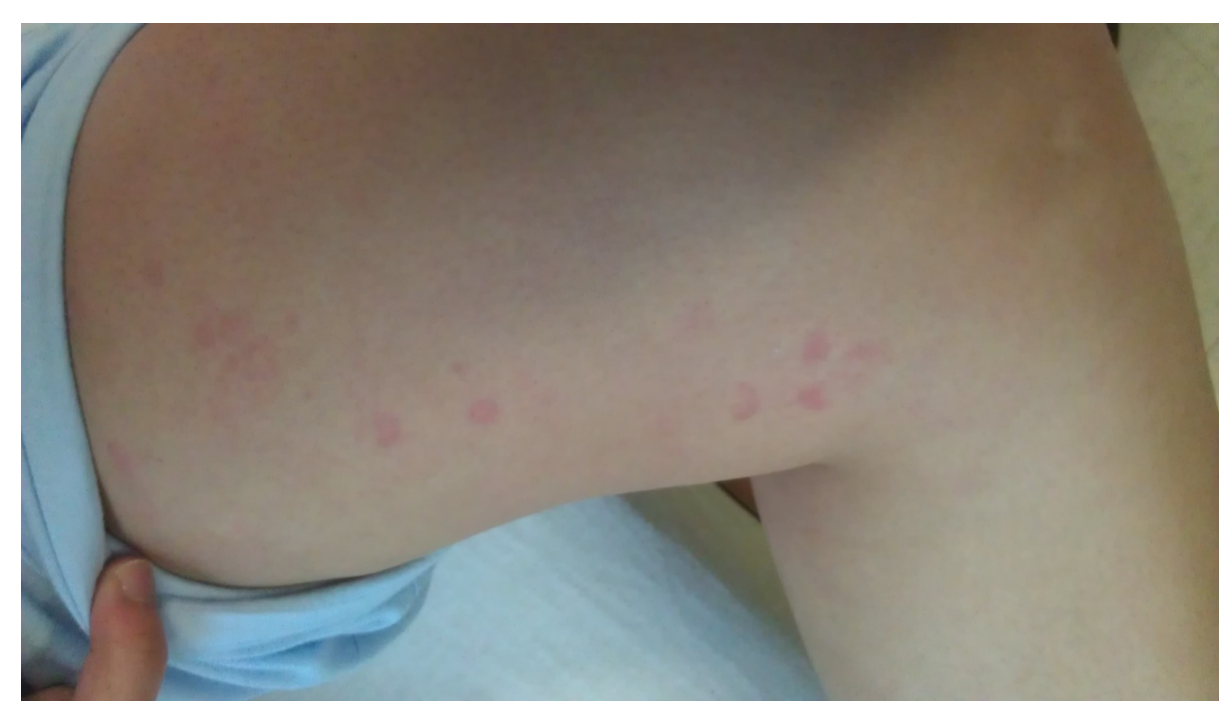

Figure 2. Rashes in the patient trunk.

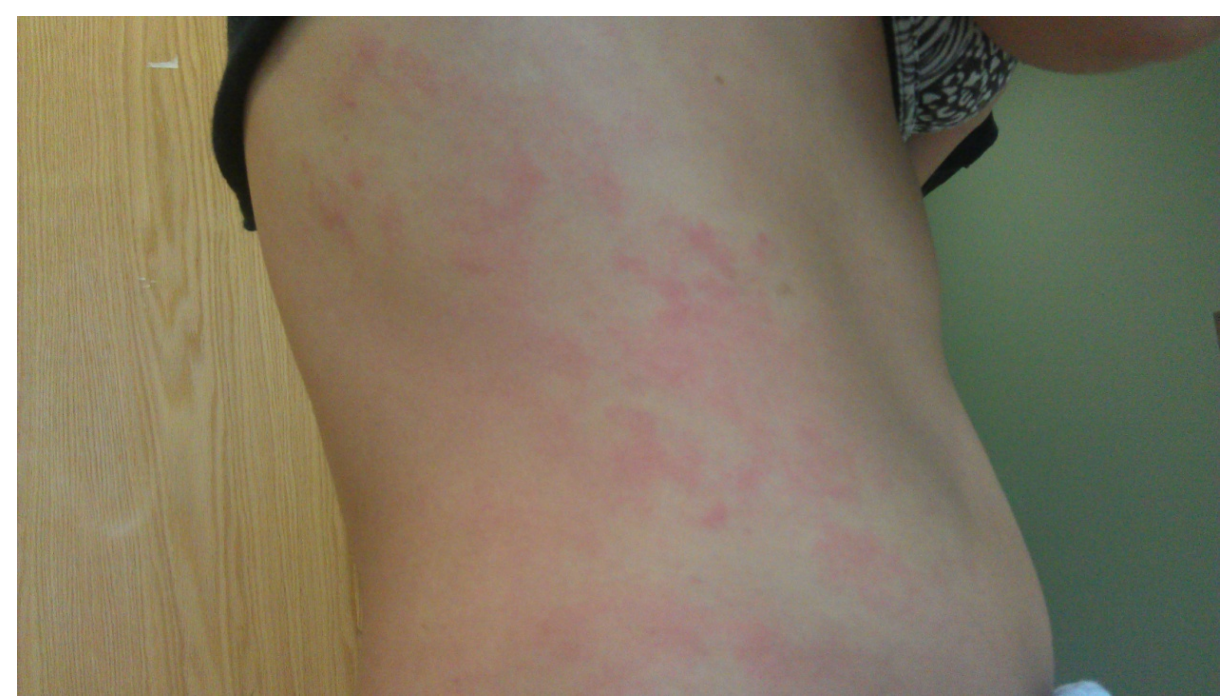

Figure 3. Rashes in medial aspect of left lower extremity. 
urticaria. Health care providers should be aware about these complications and able to medically optimize the patient as needed.

\section{REFERENCES}

[1] French, J.A., Kanner, A.M., Bautista, J., et al. (2004) Efficacy and tolerability of the new antiepileptic drugs I: Treatment of new onset epilepsy: Report of the Therapeutics and Technology Assessment Subcommittee and Quality Standards Subcommittee of the American Academy of Neurology and the American Epilepsy Society. Neurology, 62, 1252. doi:10.1212/01.WNL.0000123693.82339.FC

[2] Biton, V., Di Memmo, J., Shukla, R., et al. (2010) Adjunctive lamotrigine XR for primary generalized tonicclonic seizures in a randomized, placebo-controlled study. Epilepsy \& Behavior, 19, 352. doi:10.1016/j.yebeh.2010.07.022

[3] Bowden, C.L., Asnis, G.M., Ginsberg, L.D., et al. (2004) Safety and tolerability of lamotrigine for bipolar disorder. Drug Safety, 27, 173. doi:10.2165/00002018-200427030-00002
[4] Dichter, M.A. and Brodie, M.J. (1996) New antiepileptic drugs. The New England Journal of Medicine, 334, 15831590. doi:10.1056/NEJM199606133342407

[5] Schaub, J.E., Williamson, P.J., Barnes, E.W. and Trewby, P.N. (1994) Multisystem adverse reaction to lamotrigine (letter). Lancet, 344, 481. doi:10.1016/S0140-6736(94)91818-X

[6] Yuen, A.W. and Bihari, D.J. (1992) Multiorgan failure and disseminated intravascular coagulation in severe convulsive seizures (letter). Lancet, 340, 618. doi:10.1016/0140-6736(92)92159-D

[7] Schirop, T.H., Lufft, H., Winkler, M., et al. (1994) Bronchial mucosa reaction in Lyell-Stevens-Johnson syndrome following lamotrigine. Intensivmedizin und Notfallmedizin, 31, 343.

[8] Harchelroad, F., Lang, D. and Valeriano, J. (1994) Lamotrigine overdose. Veterinary \& Human Toxicology, 36, 372.

[9] Lofton, A.L. and Klein-Schwartz, W. (2004) Evaluation of lamotrigine toxicity reported to poison control centers. The Annals of Pharmacotherapy, 38, 1811-1815. doi:10.1345/aph.1E192 